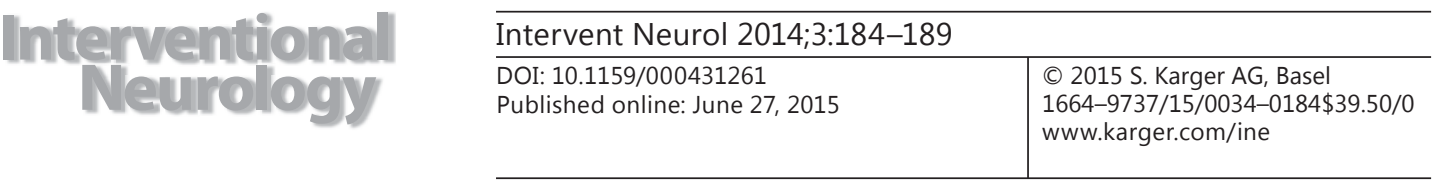

\title{
Neurointerventional Stenting and Antiplatelet Function Testing: To Do or Not to Do?
}

\author{
Tareq Kass-Hout ${ }^{a} \quad$ Yazan J. Alderazi $^{a}$ Krishna Amuluru ${ }^{a}$ Peter Jin ${ }^{a}$ \\ Carlos Ayala $^{a} \quad$ Charles Prestigiacomo ${ }^{a-c}$ Chirag D. Gandhi ${ }^{a-c}$ \\ Departments of a Neurosurgery and Neuroscience, ${ }^{b}$ Neurology and ${ }^{\mathrm{c}}$ Radiology, Rutgers \\ University School of Medicine, Newark, N.J., USA
}

\section{Key Words}

Neurointerventional procedures · Antiplatelet therapy · VerifyNow assay ·

Thrombo-embolic event

\begin{abstract}
Background and Purpose: Platelet function testing in neurointerventional (NI) procedures is still controversial. We compared the clinical outcomes between antiplatelet responders and nonresponders based on the results of the VerifyNow (VN) testing method. Methods: This is a retrospective single-center analysis of all consecutive patients who underwent NI stenting procedures from January 2007 through July 2013 and had documented preprocedural aspirin (ASA) and clopidogrel VN assays. Patients were divided into two groups based on their responsiveness to antiplatelet. Baseline characteristics, good functional outcome measured by the modified Rankin Scale (mRS) at 90 days, combined procedural complication rate defined as postprocedural stroke, in-stent thrombosis, and intraoperative rupture were compared between the two groups. Results: Our cohort included 37 patients: 26 were in the responder group (RG) and 11 were in the nonresponder group (NRG). Baseline characteristics were similar between the two groups. Even though the combined complication rate was similar between the two groups [NRG: $2 / 11$ (18\%) vs. RG: $2 / 26(7 \%) ; p=0.33$ ], there was a trend for a higher rate of good functional outcome (90-day mRS: $0-2)$ in the RG $(22 / 22,100 \%)$ as compared to the NRG $(8 / 10,80 \%)(p=0.0907)$. Conclusion: Overall, utilizing the VN antiplatelet function testing did not significantly change the clinical outcome after the NI procedures. Larger randomized trials are warranted to provide a better understanding of the utility of the antiplatelet testing in NI stenting procedures.




\section{Introduction}

Platelet function testing in neurointerventional (NI) procedures is still controversial. Even though preprocedural antiplatelet assays, theoretically, might lead to a reduced rate of thromboembolic complications, only little evidence exists supporting such practice. Light transmission aggregometry is the gold standard to test for platelet reactivity, but it is usually expensive and not technically practical, which leads to the emergence of multiple bedside assays, such as the VerifyNow (VN), that can reliably test for platelet inhibition for aspirin (ASA) and P2Y12 inhibitors. While ASA resistance is rare, clopidogrel resistance is more challenging as it is reported to be as high as $30-35 \%$ in the monitoring cohort and it is usually due, in part, to genetic variation, which is reported to increase the thromboembolic complications even with escalating the dosing of clopidogrel [1-4]. The cardiology literature representing multiple large multi-center randomized controlled trials did not show an overall clinical outcome benefit of antiplatelet therapy modification based on the preprocedural assays $[5,6]$. In the cardiology literature, the end points of complete stent thrombosis and urgent revascularization are oversimplified and should not be applied in isolation to the NI population as this population is also susceptible to other complications including perforator occlusions, symptomatic distal thromboembolism, and subarachnoid and parenchymal hemorrhages.

\section{Methods}

We conducted a retrospective review of our prospectively maintained NI database at our University Hospital from January 2007 through July 2013 to identify all NI cases that underwent cervical carotid artery stenting or any other type of intracranial stenting and had documented preprocedural ASA VN and/or P2Y12 antiplatelet assays. Patient data including demographics, antiplatelet regimen, presenting pathology, serologic testing, other medical comorbidities, and periprocedural complications (ipsilateral ischemic events, intra- or extracranial hemorrhagic events, and in-stent thrombosis) were recorded. Platelet function was assessed with the VN ASA and the VN P2Y12 assays (Accumetrics, San Diego, Calif., USA) in ASA reaction units and $\mathrm{P} 2 \mathrm{Y} 12$ reaction units (PRU), respectively.

A dual antiplatelet regimen consisting of $325 \mathrm{mg}$ ASA daily and $75 \mathrm{mg}$ clopidogrel daily, starting 7-10 days before the intervention, was given to all patients in this cohort. Even though therapeutic platelet inhibition testing remains controversial, the patients in our cohort were considered responsive if ASA reaction units $\leq 550$ and PRU $\leq 237$ [7, 8]. Patients were maintained on dual antiplatelets for 3 months after the procedure and then on ASA monotherapy indefinitely. Nonresponders to clopidogrel received intraprocedural intravenous (i.v.) eptifibatide or were loaded with $250 \mathrm{mg}$ ticlopidine before the procedure. The choice of agent was at the discretion of the neurointerventionalist. Patients who did not have documented preprocedural antiplatelet assays were excluded from the final analysis. The records of the rest of the patients were retrieved for analysis. The patients were then divided into two groups based on their preprocedural VN results into responders and nonresponders.

The primary outcome of interest was combined periprocedural complications (ipsilateral ischemic events, intra- or extracranial hemorrhagic events, in-stent thrombosis). The secondary outcomes of interest in the two groups included the good functional outcome defined by modified Rankin Scale (mRS) scores of 0-2 at 90 days after the procedure. IBM SPSS software version 20 was utilized to perform the desired statistical analysis. A simple two-sample t test was performed to compare the age. The $\chi^{2}$ test of independence was used to compare the rest of the variables between the two groups.

The Institutional Review Board at Rutgers University approved this retrospective study. 
Table 1. Demographic and clinical characteristics in the RG and NRG

\begin{tabular}{lccl}
\hline Characteristics & RG $(\mathrm{n}=26)$ & NRG $(\mathrm{n}=11)$ & $\mathrm{p}$ value \\
\hline Mean age \pm SD, years & $53.4 \pm 15.1$ & $59.9 \pm 11.1$ & 0.20 \\
Female gender & $16(46.1)$ & $8(72.7)$ & 0.514 \\
Hypertension & $11(42.3)$ & $7(63.6)$ & 0.23 \\
Diabetes mellitus & $4(15)$ & $1(9)$ & 0.608 \\
Dyslipidemia & $5(19.2)$ & $4(36.3)$ & 0.78 \\
Current smoker & $6(23)$ & $3(27.2)$ & 0.579 \\
Coronary artery disease & $2(7.6)$ & $4(36.3)$ & 0.094 \\
Previous stroke & $6(23)$ & $4(36.3)$ & 0.405 \\
\hline
\end{tabular}

Values are presented as n (\%) unless otherwise indicated.

Table 2. Comparison of the primary and secondary outcomes between the RG and NRG

\begin{tabular}{lllc}
\hline Characteristics & RG (n=26) & NRG (n=11) & p value \\
\hline $\begin{array}{l}\text { Good functional outcome (90-day mRS: 0-2) } \\
\text { Combined procedural complication (stroke, in-stent }\end{array}$ & $22 / 22(100)$ & $8 / 10(80)$ & 0.0907 \\
$\quad$ & $2(7)$ & $2(18)$ & 0.33 \\
$\quad$ thrombosis, and intraoperative rupture) & $0(0)$ & $2(18)$ & 0.083 \\
Postoperative stroke & $1(3)$ & $0(0)$ & 0.85 \\
In-stent thrombosis & $1(3)$ & $0(0)$ & 0.85 \\
Intraoperative aneurysm rupture & & & \\
\hline
\end{tabular}

Values are presented as n (\%).

\section{Results}

\section{Clinical and Treatment Characteristics}

A total of 51 patients underwent extra- or intracranial stenting and were included in the study. Only 37 of them had preprocedural antiplatelet assays utilizing the VN test. Among those, 11 patients were nonresponders to Plavix (nonresponder group, NRG) and 1 of them was also a nonresponder to ASA. Out of these, 8 patients received i.v. eptifibatide, and 3 patients received i.v. ticlodipine intraprocedurally. In the NRG, 5 patients had extra-cranial stenting with Acculink stents (Abbott Vascular, Santa Clara, Calif., USA), and 6 patients had intracranial stenting [4 had stent-assisted coiling with Enterprise stent (Codman, Raynham, Mass., USA), 1 had a basilar Wingspan stent (Stryker, Kalamazoo, Mich., USA), and 1 had a pipeline stent (Covidien Vascular Therapies, Mansfield, Mass., USA)]. The other 26 patients were in the responder group (RG). In the RG, 14 patients had extra-cranial stenting [12 cervical carotid stents with 10 Acculink stents (Abbott Vascular) and 2 vertebral stents: 1 Xience stent (Abbott Vascular) and 1 PROMUS stent (Boston Scientific, Natick, Mass., USA)], and 12 patients had intracranial stenting ( 6 patients had stent-assisted coiling, 3 had atherosclerotic stenting, and 3 had a pipeline). The mean patient age in the NRG was $59.9 \pm 11.1$ years, which was similar to the mean age in the $R G$, which was $53.4 \pm 15.1$ years $(p=0.20)$. The percentage of women was also similar in the two groups (72.7 vs. $46.1 \%$, respectively; $\mathrm{p}=0.514$ ). Risk factors for stroke were similar in the two groups (table 1). 


\section{Primary and Secondary Clinical Outcomes}

The total procedural complication rate (primary end point) was similar across the two groups [NRG: $2 / 11(18 \%)$ vs. RG: $2 / 26(7 \%) ; p=0.33)$. There were similar rates of in-stent thrombosis [NRG: 0/11 (0\%) vs. RG: $1 / 26(3 \%) ; p=0.85$ ] and intraoperative aneurysm rupture [NRG: $0 / 11(0 \%)$ vs. RG: $1 / 26(3 \%) ; p=0.85]$ across both groups. However, the NRG had a trend towards a higher chance of postoperative stroke [NRG: 2/11 (18\%) vs. RG: 0/26 $(0 \%) ; p=0.083$; table 2]. Among the 37 patients, only 32 had their functional assessment at 90 days at the time of the analysis (10 patients in the NRG and 22 patients in the RG). There was a trend for a higher rate of good functional outcome (90-day mRS: $0-2$ ) in the RG (22/22, $100 \%)$ as compared to the NRG $(8 / 10,80 \%)(p=0.0907$; table 2$)$.

\section{Discussion}

Our analysis adds to the scant evidence of using platelet function tests in NI procedures. Even though our study showed a better overall functional outcome and decreased rates of postoperative stroke in the RG, this might not be completely explained by the antiplatelet responsiveness. This is particularly true given the small number of patients and the pathophysiological diversity of our cohort. While there has been more cardiac and neurological literature to suggest that patients who are resistant to clopidogrel have a higher rate of periprocedural thrombotic complications [3], there is no solid evidence for routine utility of platelet function tests for patients undergoing NI procedures. The only two randomized clinical trials to look at this were both found in the cardiology literature. In the GRAVITAS trial, resistant patients were randomized to receiving either standard or double-dose clopidogrel after percutaneous coronary intervention [6]. There was no difference in the development of death, myocardiac infarction, or stent thrombosis between the two groups. However, patients were given double-dose clopidogrel and not targeted to a specific PRU level. In the ARCTIC trial, patients with percutaneous coronary intervention were given additional clopidogrel loading or were switched to prasugrel. Again, there was no difference in outcomes. However, PRU $<230$ were not achieved in $15 \%$ of patients on follow-up testing. As mentioned above, the demographics and risk factors differ among NI and cardiovascular patients. The clinically significant risk of cardiovascular stenting is largely stent thrombosis, whereas in NI patients, hemorrhagic and thromboembolic complications are serious concerns. While these are important differences, lessons can be learned from the similarities in the data. The rates of platelet activity in neurovascular patients on antiplatelet medications have been found to be similar to those in cardiovascular patients $[9,10]$. A previous report encompassing 48 procedures with a pipeline embolization device found that a PRU value of $>240$ predicted thromboembolic events [11]. PRU values $<198$ were found to have a protective effect against ischemic events in an observational carotid stenting study [4]. Furthermore, reports suggest that a PRU value $<60$ is associated with an increased hemorrhagic risk [11]. There are limited data in regard to neurovascular patients and modification of treatment. A recent prospective study found a nonsignificant decrease in thromboembolic complications with double dose for patients with $\leq 20 \%$ platelet inhibition [3]. Optimal target PRU values for NI procedures have yet to be determined as there is a paucity of high-quality studies specific to NI procedures [12].

In our cohort, 11 patients were found to be clopidogrel resistant and were given either ticlodipine or eptifibatide. Two of these patients had postoperative stroke. Several reports have shown that platelet inhibition can be increased through different methods in resistant patients. Increasing doses of ASA or clopidogrel may reduce resistance in some patients. In addition, switching to a different antiplatelet agent can also reduce platelet activity. In pa- 
tients resistant to clopidogrel, double dosing has been shown to result in therapeutic platelet activity in some patients [13]. Nevertheless, this reduction in platelet activity has not decreased thrombotic complications. Also, the overall complication rate in our cohort was similar to what has previously been reported in NI procedures that did not use antiplatelet assays.

As this was a retrospective study, we did not have a set protocol for the universal testing of patients undergoing NI stenting procedures and for modifications of antiplatelet therapy based on non- or hyper-responders. Moreover, we were not able to identify the reasons for preferring either eptifibatide or ticlopidine in the medical records. In our cohort, these medications were used for high platelet activity on antiplatelet testing rather than intraprocedural thrombus. Our study has significant limitations including its retrospective design and relatively small sample size, in addition to the pathophysiological diversity of the cohort. However it calls into question important aspects of NI stenting procedures and sheds light on the importance of validating the newer antiplatelet function testing.

\section{Conclusion}

Overall, the utilization of the VN antiplatelet function testing did not significantly change the clinical outcome after NI procedures. This echoes the scant literature that advocates against the use of antiplatelet assays for the general population undergoing such procedures. Larger randomized trials are warranted to provide a better understanding of the utility of antiplatelet testing in NI stenting procedures.

\section{Disclosure Statement}

There is no financial relationship and no conflict of interest directly in conjunction with this work.

\section{References}

1 Goh C, Churilov L, Mitchell P, Dowling R, Yan B: Clopidogrel hyper-response and bleeding risk in neurointerventional procedures. AJNR Am J Neuroradiol 2013;34:721-726.

-2 Prabhakaran S, Wells KR, Lee VH, Flaherty CA, Lopes DK: Prevalence and risk factors for aspirin and clopidogrel resistance in cerebrovascular stenting. AJNR Am J Neuroradiol 2008;29:281-285.

-3 Fifi JT, Brockington C, Narang J, Leesch W, Ewing SL, Bennet H, et al: Clopidogrel resistance is associated with thromboembolic complications in patients undergoing neurovascular stenting. AJNR Am J Neuroradiol 2013; 34:716-720.

-4 Sorkin GC, Dumont TM, Wach MM, Eller JL, Mokin M, Natarajan SK, et al: Carotid artery stenting outcomes: do they correlate with antiplatelet response assays? J Neurointervent Surg 2014;6:373-378.

5 Collet JP, Cuisset T, Rangé G: Bedside monitoring to adjust antiplatelet therapy for coronary stenting. N Engl J Med 2012;367:2100-2109.

-6 Price MJ, Berger PB, Teirstein PS: Standard- vs high-dose clopidogrel based on platelet function testing after percutaneous coronary intervention: the GRAVITAS randomized trial. JAMA 2011;305:1097-1105.

-7 Nielsen HL1, Kristensen SD, Thygesen SS, Mortensen J, Pedersen SB, Grove EL, Hvas AM: Aspirin response evaluated by the VerifyNow Aspirin System and light transmission aggregometry. Thromb Res 2008;123: 267-273.

8 Marcucci R, Gori AM, Paniccia R, Giusti B, Valente S, Giglioli C, Buonamici P, Antoniucci D, Abbate R, Gensini GF: Cardiovascular death and nonfatal myocardial infarction in acute coronary syndrome patients receiving coronary stenting are predicted by residual platelet reactivity to ADP detected by a point-of-care assay: a 12-month follow-up. Circulation 2009;119:237-242.

-9 Lee DH AA, Morsi H, Shaltoni H: Dual antiplatelet therapy monitoring for neurointerventional procedures using a point-of-care platelet function test: a single-center experience. AJNR Am J Neuroradiol 2008;29:13891394. 
10 Pandya DJ, Fitzsimmons BFM, Wolfe TJ, Hussain SI, Lynch JR, Ortega-Gutierrez S, et al: Measurement of antiplatelet inhibition during neurointerventional procedures: the effect of antithrombotic duration and loading dose. J Neuroimaging 2010;20:64-69.

$\checkmark 11$ Delgado Almandoz JE, Crandall BM, Scholz JM, Fease JL, Anderson RE, Kadkhodayan Y, et al: Pre-procedure P2Y12 reaction units value predicts perioperative thromboembolic and hemorrhagic complications in patients with cerebral aneurysms treated with the Pipeline Embolization Device. J Neurointerv Surg 2013;5(suppl 3): iii3-iii10.

12 Gandhi CD, Bulsara KR, Fifi J, Kass-Hout T, Grant RA, Delgado Almandoz JE, English J, Meyers PM, Abruzzo T, Prestigiacomo CJ, Powers CJ, Lee SK, Albani B, Do HM, Eskey CJ, Patsalides A, Hetts S, Hussain MS, Ansari SA, Hirsch JA, Kelly M, Rasmussen P, Mack W, Pride GL, Alexander MJ, Jayaraman MV; SNIS Standards and Guidelines Committee: Platelet function inhibitors and platelet function testing in neurointerventional procedures. J Neurointerv Surg 2014;6:567-577.

13 Mega JL, Hochholzer W, Frelinger AL: Dosing clopidogrel based on cyp2c19 genotype and the effect on platelet reactivity in patients with stable cardiovascular disease. JAMA 2011;306:2221-2228. 